\title{
How Are Systematic Reviews of Prevalence Conducted? A Methodological Study
}

Celina Borges Migliavaca ( $\nabla$ celinabm7@gmail.com )

Universidade Federal do Rio Grande do Sul https://orcid.org/0000-0003-1389-2311

\section{Cinara Stein}

Hospital Moinhos de Vento

\section{Verônica Colpani}

Hospital Moinhos de Vento

\section{Timothy Hugh Barker}

The University of Adelaide Joanna Briggs Institute

\section{Zachary Munn}

The University of Adelaide Joanna Briggs Institute

\section{Maicon Falavigna}

Hospital Moinhos de Vento

\section{Research article}

Keywords: prevalence, systematic review, methodological quality, meta-epidemiological study

Posted Date: January 6th, 2020

DOl: https://doi.org/10.21203/rs.2.20036/v1

License: (c) (i) This work is licensed under a Creative Commons Attribution 4.0 International License. Read Full License

Version of Record: A version of this preprint was published at BMC Medical Research Methodology on April 26th, 2020. See the published version at https://doi.org/10.1186/s12874-020-00975-3. 


\section{Abstract}

Background There is a notable lack of methodological and reporting guidance for systematic reviews of prevalence data. This information void has the potential to result in reviews that are inconsistent and inadequate to inform healthcare policy and decision making. The aim of this meta-epidemiological study is to describe the methodology of recently published prevalence systematic reviews.

Methods We searched MEDLINE (via PubMed) from February 2017 to February 2018 for systematic reviews of prevalence studies. We included systematic reviews assessing the prevalence of any clinical condition using patients as the unit of measurement and we summarized data related to reporting of and methodology of the reviews.

Results A total of 235 systematic reviews of prevalence were analyzed. The median number of authors was 5 (interquartile range [IQR] 4-7), the median number of databases searched was 4 (3-6) and the median of studies included in each review was 24 (IQR 15-41.5). Search strategies were presented for $68 \%$ of reviews. Forty five percent of reviews received external funding, and $24 \%$ did not provide funding information. Twenty three percent of included reviews had published or registered the systematic review protocol. Reporting guidelines were used in $72 \%$ of reviews. The quality of included studies was assessed in $80 \%$ of reviews. Only 12 reviews assessed the overall quality of evidence ( $50 \%$ of them using GRADE). Meta-analysis was conducted in $65 \%$ of reviews; only $1 \%$ used Bayesian methods. Random effect metaanalysis was used in $94 \%$ of reviews; among them, $75 \%$ did not report the variance estimator used. Among the reviews with meta-analysis, $70 \%$ did not report how data was transformed; $59 \%$ percent conducted subgroup analysis, $38 \%$ conducted meta-regression and $2 \%$ estimated prediction interval; 12 was estimated in $95 \%$ of analysis. Publication bias was examined in $48 \%$. The most common software used was STATA (55\%).

Conclusions Our results indicate that there are significant inconsistencies regarding how these reviews are conducted. Many of these differences arose in the assessment of methodological quality and the formal synthesis of comparable data. This variability indicates the need for clearer reporting standards and consensus on methodological guidance for systematic reviews of prevalence data.

\section{Background}

The proportion of a population currently suffering from a disease or particular condition of interest (prevalence) is an important (1) metric that allows researchers to assess disease burden, that is, who among the population is experiencing a certain disease, at a very specific point in time, typically measured using a cross-sectional study design. (2) The subsequent synthesis of this information in the form of a rigorously conducted and transparently reported systematic review has significant potential to better inform social and healthcare professionals, policy makers and consumers to better manage and plan for this disease burden.(3) 
The number of systematic reviews of prevalence data has increased steadily over the last decade. (4) As can be seen in Figure 1, a search of PubMed, conducted in October 2019 using the terms "systematic review" and "prevalence" in the title, identified an almost ten-fold increase in the number of reviews published from 2006 to 2017.

Figure 1: Number of systematic reviews of prevalence indexed in PubMed between 1998 and 2018

Despite this uptake and increased interest in the scientific community in the conduct of a systematic review of prevalence data, there remains little discourse on how these reviews should be conducted and reported. Although methodological guidance from the Joanna Briggs Institute exists for these review types, (5) there currently appears to be little other discussion on how these reviews should be prepared, performed and reported. This is an important consideration, as standards of reporting and conduct for systematic reviews of traditional systematic reviews (i.e. systematic reviews of interventions) (6) are considered commonplace, and even endorsed by journal editors. Such standards and guidelines have not been as readily developed or adopted for systematic reviews of prevalence data. This is despite the fact that there is now general acceptance that there needs to be different types of approaches for systematic reviews looking at different types of evidence. $(6-8)$

This lack of accepted, readily adopted and easy to implement methodological and reporting guidance for systematic reviews of prevalence data has the potential to result in inconsistent, varied, inadequate and potentially inappropriate research conduct. Due to the particular importance of this kind of data in enabling health researchers and policy makers to quantify disease amongst populations, such unregulated research conduct is likely to have far-reaching and complicated consequences for the wider public community.

As such, the objective of this research project was to conduct a meta-epidemiological review of all systematic reviews that have been published in peer-reviewed journals and evaluated a question regarding the prevalence of a certain disease, symptom or condition. This project will allow us to investigate how these reviews are conducted and the discrepancies in methods used, to provide an overview of all methods utilized by systematic reviews authors asking a question of prevalence. The results of this project can potentially inform the development of future guidance for this review type.

\section{Methods}

\section{Search strategy:}

To retrieve potentially relevant reviews, we searched MEDLINE (via PubMed) using the terms 'systematic review' and 'prevalence' in the title (prevalence[TI] AND "systematic review"[TI] AND ("2017/02/01"[PDAT]: "2018/02/01"[PDAT])).. The search was limited to studies published between February 2017 and February 2018. 


\section{Study selection:}

The selection of studies was conducted in two phases by two independent reviewers. First, we screened titles and abstracts. Then, we retrieved the full-text of potentially relevant studies to identify studies meeting our inclusion criteria. We included systematic reviews of prevalence of any clinical condition. We excluded primary studies, letters, narrative reviews, systematic reviews of interventions or diagnostic accuracy and systematic reviews of prevalence that did not use patients as the unit of measurement, as well as studies not published in English.

\section{Data abstraction}

Using a standard and piloted form, one author extracted relevant data from each review and another author independently checked all data. Discrepancies were discussed and solved by consensus or by a third reviewer.

We abstracted the following data from individual studies: general information about the paper (number of authors, journal and year of publication), reporting of funding, reporting of search strategy, number and description of databases consulted, number of reviewers involved in each step of the review, number of studies included in the review, methods for risk of bias appraisal and quality of evidence assessment, synthesis of results, assessment of publication bias and details of meta-analytic processes if metaanalysis was conducted (including variance estimator, transformation of data, heterogeneity assessment, and software used).

\section{Data analysis}

Results are presented using descriptive statistics. Quantitative variables are presented as means and standard deviations or median and interquartile range, as appropriate; and qualitative variables are presented in absolute and relative frequencies.

\section{Results}

Our search resulted in 325 articles. We assessed the full text of 251 and included 235 of them in our analysis. Figure 2 presents the flowchart of study selection. The main characteristics of the included studies are described in Table 1. The complete list of included studies is presented in Additional file 1 and the list of full text excluded with reasons is presented in Additional file 2. The complete data extraction table is presented in Additional file 3.

\section{Figure 2: Flowchart of study selection}

Of 235 studies included, 83 (35.3\%) presented only narrative synthesis, while 152 (64.7\%) performed meta-analysis. These 235 articles were published in 85 different journals. 
Fifty-three studies (22.6\%) published or registered a protocol, and $170(72.3 \%)$ used a reporting guideline, including PRISMA ( $n=161,68.5 \%)$, MOOSE ( $n=27,11.5 \%)$ and GATHER ( $n=2,0.9 \%)$. In 56 studies (45.1\%) there was no reporting of funding.

The median number of databases searched in each review was 4 (IQR 3-6); 231 studies (98.3\%) used PubMed, 146 (62.1\%) used Embase, 93 (39.6\%) used Web of Science, and 70 (29.8\%) used Cochrane CENTRAL, even though this is a database focused on interventional studies and systematic reviews. Most reviews ( $n=228,97.0 \%$ ) reported the search strategy used, although 69 (29.4) reported it incompletely. The median number of included original studies in each systematic review was 24 (IQR 1541.5).

One hundred and eighty-eight reviews (80.0\%) assessed the methodological quality of included studies. One hundred and five (44.7\%) reported that this assessment was performed by at least two reviewers. There was a great variability regarding the instruments used to critically appraise included studies. Twenty-four studies (10.2\%) developed new tools, and 24 (10.2\%) used non-validated tools from previous similar systematic reviews. Among validated and specific tools to assess prevalence studies, the Joanna Briggs Institute (JBI) prevalence critical appraisal tool was the most used $(n=21,8.9 \%)$. Fifteen reviews (6.4\%) used STROBE, a reporting guideline, to assess the methodological quality of included studies. Thirty-two reviews (17.0\%) used assessment of methodological quality as an inclusion criterion for the review.

Only 9 studies (3.8\%) assessed the quality of the body of evidence, with GRADE being the highest cited methodology $(n=4,1.7 \%)$. Not all methods used to assess the quality of evidence were validated and appropriate. For instance, one study summarized the quality of evidence as the mean STROBE score of included studies.

\section{Statistical methods for meta-analysis}

One hundred and fifty-two studies (64.7\%) conducted meta-analysis to summarize prevalence estimates. The methods used by these reviews are summarized in Table 2 .

The vast majority of studies ( $n=151,99.3 \%)$ used classic methods instead of Bayesian approaches to pool prevalence estimates. The majority of studies pooled estimates using a random effects model $(n=141,93.4 \%)$, and only 7 studies (4.6\%) utilized the fixed effect model. Two studies (1.3\%) used the 'quality model', where the weight of each study was calculated based on a quality assessment. However, both reviews used non-validated methods to critically appraise included studies.

In relation to variance estimation in the reviews that conducted random effects meta-analysis, the DerSimonian and Laird method was used in 30 reviews (21.3\%). However, 106 studies $(75.2 \%)$ did not report the variance estimator used. Only 45 studies (29.6\%) reported how they transformed the prevalence estimates, and the most used methods were Freeman-Tukey double arcsine $(n=32,21.1 \%)$, logit $(n=5$, $3.3 \%)$ and $\log (n=4,2.6 \%)$. Heterogeneity among studies was assessed with the $\mathrm{I}^{2}$ statistics in 114 studies 
(94.7\%), meta-regression in 57 studies (37.5\%) and subgroup analysis was performed in 89 studies (58.6\%). Most analyses ( $n=105,76.1 \%)$ had an $\mathrm{I}^{2}$ estimate of $90 \%$ or more. Publication bias was assessed with funnel plots $(n=56,36.8 \%)$ and Egger's test $(n=54,35.5 \%)$. Prediction interval was only estimated in 3 reviews (2.0\%).

\section{Table 1: Main characteristics of included systematic reviews $(n=235)$}

\section{Table 2: Methods used for meta-analysis $(n=152)$}

\section{Discussion}

This meta-epidemiological study identified 235 systematic reviews addressing a question related to the prevalence of any clinical condition. Our investigations have found that across the body of systematic reviews of prevalence data, there are significant and important discrepancies in how these reviews consider searching, risk of bias and data synthesis.

One area for potential guidance to inform future systematic reviews of prevalence data is in the risk of bias or critical appraisal stage. As can be seen from the results of our review, there are a number of checklists and tools that have been used. Interestingly, some of these tools have been designed specifically for studies reporting prevalence information, whilst some other tools have been adapted for the purpose. Some of the tools utilized were not appropriate for this assessment, such as the Newcastle Ottawa Scale (designed for observational studies)(7) or STROBE (a reporting standard)(8) as a quality assessment tool. This is particularly concerning when reviews have used results of quality assessment to determine inclusion in the review, as was the case in $17 \%$ of the included studies. The combination of (a) using inadequate or inappropriate critical appraisal tools and (b) using the results of these tools to decide upon inclusion in a systematic review could lead to the inappropriate exclusion of relevant studies, which can alter the final results and produce misleading estimates. As such, there is an urgent need for the development and validation of a tool for assessing prevalence, along with endorsement and acceptance by the community to assist with standardization in this field. In the meantime, we urge reviewers to refer to the JBI critical appraisal tool,(4) which has been formally evaluated and is increasingly used across these types of reviews.

Encouragingly, $72.3 \%$ of the included reviews adhered to or cited a reporting guideline in their review. The main reporting guideline reported was the PRISMA statement.(6) However, this reporting guideline was designed particularly for reviews of interventions of therapies. As such, there have been multiple extensions to the original PRISMA statement for various review types $(9,10)$, yet no extension has yet been considered for systematic reviews of prevalence data. To ensure there is a reporting standard for use in prevalence systematic reviews, an extension of the PRISMA statement for this review type is recommended.

It was encouraging to see that multiple databases were often searched during the systematic review process, which is a recommendation for all review types. However, it is also important in systematic 
reviews that all the evidence is identified, and in the case of prevalence information, it may be particularly useful to search for data in unpublished sources, such as clinical registries, government reports, census data, and national administrative datasets for example.(11,12) Further guidance on searching for evidence in prevalence reviews is required.

In our review, we found $64.7 \%$ of reviewers conducted meta-analysis. There has been debate regarding the appropriateness of meta-analysis within systematic reviews of prevalence, $(13,14)(15-17)$ largely surrounding whether synthesizing across different populations is appropriate, as we reasonably expect prevalence rates to vary across different contexts and where different diagnostic criteria may be employed. However, meta-analysis, when done appropriately, can provide important information regarding the burden of disease, including identifying differences amongst populations and regions, changes over time, and can provide a summarized estimate that can be used when calculating baseline risk, such as in GRADE summary of findings tables. Further investigation and guidance of when metaanalysis should be considered and when it is unlikely to be beneficial in prevalence reviews is required.

The vast majority of meta-analyses used classic methods and a random effects model, which is appropriate in these types of reviews (16) (17). Although the common use of random effects across the reviews is encouraging, this is where the consistency ends, as we once again see considerable variability in the choice of methods to transform prevalence estimates for proportional meta-analysis. The most widely used approach was the Freeman-Tukey double arscine transformation. This has been recommended as the preferred methods for transformation $(14,17)$, although more recently it has come under question.(18) As such, further guidance and investigation into meta-analytical techniques is urgently required.

In reviews including meta-analysis, heterogeneity was assessed with the $\mathrm{I}^{2}$ in $94.7 \%$ of the included studies. Although $\mathrm{I}^{2}$ provides a useful indication of statistical heterogeneity amongst studies included in a meta-analysis, it can be misleading in cases where studies are providing large datasets with precise confidence intervals (such as in prevalence reviews).(19) Other assessments of heterogeneity, such as $\mathrm{T}^{2}$, may be more appropriate in these types of reviews. $(19,20)$ Further guidance on assessing heterogeneity in these types of reviews is required.

Of the 235 systematic reviews analyzed, only 9 (3.8\%) included a formal quality assessment or process to establish certainty of the entire body of evidence. Separate to critical appraisal, quality assessment of the entire body of evidence considers other factors in addition to methodological assessment that may impact on the subsequent recommendations drawn from such evidence.(21) Of these 9 reviews, only 4 $(1.7 \%)$ followed the GRADE approach (22) which is now the commonplace methodology to reliably and sensibly rate the quality of the body of evidence. The considerably small number of identified reviews that included this formal quality assessment of the entire body of evidence might be linked to the lack of formal guidance from the GRADE working group on how to apply GRADE in systematic reviews of prevalence data. However, there is some guidance into the use of GRADE for baseline risk or overall prognosis (23) which may be useful for these types of reviews. Whilst the number of identified studies 
that utilized GRADE in particular is small, it is encouraging to see the adoption of these methods in systematic reviews of prevalence data, as these examples will help contribute to the design of formal guidance for this data type in future.

It is reasonable to expect some differences in how these types of reviews are conducted, as different authors groups will rationally disagree regarding key issues, such as whether Bayesian approaches are best or the ideal method for transforming data. However, the wide inconsistency and variability noted in our review are far beyond the range of what could be considered reasonable and is of considerable concern for a number of reasons. First (1); this lack of standardization may encourage unnecessary duplication of reviews as reviewers approach similar questions with their own preferred methods; (2) novice reviewers searching for exemplar reviews may follow inadequate methods as they conduct these reviews; (3) the general confusion in end users, peer reviewers and readers of these reviews as they are required to become accustomed to various ways of conducting these studies; (4) review authors themselves following inadequate approaches, missing key steps or information sources, and importantly increasing the potential for review authors to report inaccurate or misleading summarized estimates; (5) a lack of standardization across reviews limits the ability to streamline, automate or use artificial intelligence to assist systematic review production, which is a burgeoning field of inquiry and research; and (6) these poorly conducted and reported reviews may have limited or even detrimental impacts on the planning and provision of healthcare. As such, we urgently call for the following to occur to rectify these issues, (1); further methods development in this field and for updated guidance on the conduct of these types of reviews, (2); we urgently require a reporting standard for these reviews (such as an extension to PRISMA), (4); the development (or endorsement) of a tailored risk of bias tool for studies reporting prevalence estimates, (5); the further development and promotion of software(24) and training materials(25) for these review types to support authors conducting these reviews.

\section{Limitations of our study}

In this study, we have collated and interrogated the largest dataset of systematic reviews of prevalence currently available. Although only a sample, it is likely to be representative of all published prevalence systematic reviews, although it is important that we acknowledge we only searched MEDLINE over a period of 1 year. There is potential that systematic reviews of prevalence published in journals not indexed (or not published at all) are meaningfully different from those characterized in our results. However, given that reviews indexed on MEDLINE are (hypothetically) likely to be of higher quality than those not indexed, and given that we still identified substantial inconsistency, variability and potentially inappropriate practices in this sample, we doubt that a broader search will have altered our main conclusions significantly.

\section{Conclusions}


This meta-epidemiological review found that of all published systematic reviews, there are considerable discrepancies in terms of conduct, reporting, risk of bias assessment and data synthesis. This variability is understandable given the limited amount of guidance in this field, the lack of a reporting standard and a widely accepted risk of bias or critical appraisal tool. The results of this review can be used to inform future reviewer's conducting systematic reviews of prevalence, but more importantly our findings are a call to action to the evidence synthesis community to develop guidance and reporting standards urgently for these types of systematic reviews.

\section{List Of Abbreviations}

AHCPR: Agency for Health Research and Quality

GATHER: Guidelines for Accurate and Transparent Health Estimates Reporting

GRADE: Grading of Recommendations Assessment, Development and Evaluation

IQR: interquartile range

JBI: Joanna Briggs Institute

MOOSE: Meta-Analysis of Observational Studies in Epidemiology

PRISMA: Preferred Reporting Items for Systematic Reviews and Meta-Analyses

PROSPERO: International Prospective Register of Systematic Reviews

\section{Declarations}

Ethics approval and consent to participate: Not applicable.

Consent for publication: Not applicable.

Availability of data and materials: All data generated or analysed during this study are included in this published article and its supplementary information files.

Competing interests: The authors declare that they have no competing interests.

Funding: This research did not receive any specific grant from funding agencies in the public, commercial, or not-for-profit sectors.

Authors' contributions: MF conceived the study. CBM, CS and VC conducted study selection, data extraction and data analysis. VC, MF and ZM supervised the conduction of study. All authors discussed the concepts of this manuscript and interpreted the data. CBM and THB wrote the first version of the manuscript. All authors made substantial revisions and read and approved the final manuscript. 
Acknowledgements: Not applicable.

\section{References}

1.Fletcher RH, Fletcher SW, Fletcher GS. Clinical epidemiology: the essentials: Lippincott Williams \& Wilkins; 2012.

2.Coggon D, Barker D, Rose G. Epidemiology for the Uninitiated: John Wiley \& Sons; 2009.

3.Munn Z, Moola S, Riitano D, Lisy K. The development of a critical appraisal tool for use in systematic reviews addressing questions of prevalence. International journal of health policy and management. 2014;3(3):123.

4.Munn Z, Moola S, Riitano D, Lisy K. The development of a critical appraisal tool for use in systematic reviews addressing questions of prevalence. Int J Health Policy Manag. 2014;3(3):123-8.

5.Munn Z, Moola S, Lisy K, Riitano D, Tufanaru C. Systematic reviews of prevalence and incidence. In: Aromataris E, Munn Z, editors. Joanna Briggs Institute Reviewer's Manual: The Joanna Briggs Institute; 2017.

6.Liberati A, Altman DG, Tetzlaff J, Mulrow C, Gotzsche PC, loannidis JP, et al. The PRISMA statement for reporting systematic reviews and meta-analyses of studies that evaluate healthcare interventions: explanation and elaboration. BMJ (Clinical research ed). 2009;339:b2700.

7.Wells G, Shea B, O'connell D, Peterson J, Welch V, Losos M, et al. The Newcastle-Ottawa Scale (NOS) for assessing the quality of nonrandomised studies in meta-analyses. Ottawa (ON): Ottawa Hospital Research Institute; 2009. Available in March. 2016.

8.Von Elm E, Altman DG, Egger M, Pocock SJ, Gøtzsche PC, Vandenbroucke JP. The Strengthening the Reporting of Observational Studies in Epidemiology (STROBE) statement: guidelines for reporting observational studies. Annals of internal medicine. 2007;147(8):573-7.

9.Tricco AC, Lillie E, Zarin W, O'Brien KK, Colquhoun H, Levac D, et al. PRISMA extension for scoping reviews (PRISMA-ScR): checklist and explanation. Annals of internal medicine. 2018;169(7):467-73.

10.McInnes MD, Moher D, Thombs BD, McGrath TA, Bossuyt PM, Clifford T, et al. Preferred reporting items for a systematic review and meta-analysis of diagnostic test accuracy studies: the PRISMA-DTA statement. Jama. 2018;319(4):388-96.

11.Munn Z, Moola S, Lisy K, Riitano D. The synthesis of prevalence and incidence data. Pearson A, editor. Philadelphia: Lippincott Williams \& Wilkins; 2014.

12.lorio A, Stonebraker JS, Chambost H, Makris M, Coffin D, Herr C, et al. Establishing the Prevalence and Prevalence at Birth of Hemophilia in Males: A Meta-analytic Approach Using National RegistriesMale 
Prevalence and Prevalence at Birth of Hemophilia. Annals of Internal Medicine. 2019.

13.Rao SR, Graubard BI, Schmid CH, Morton SC, Louis TA, Zaslavsky AM, et al. Meta-analysis of survey data: application to health services research. Health Services and Outcomes Research Methodology. 2008;8(2):98-114.

14.Barendregt JJ, Doi SA, Lee YY, Norman RE, Vos T. Meta-analysis of prevalence. Journal of epidemiology and community health. 2013;67(11):974-8.

15.Jablensky A. Schizophrenia: the epidemiological horizon. In: Hirsch S, Weinberger D, editors. Schizophrenia. Oxford:: Black-well Science; 2003. p. 203-31.

16.Saha S, Chant D, McGrath J. Meta-analyses of the incidence and prevalence of schizophrenia: conceptual and methodological issues. International journal of methods in psychiatric research. 2008;17(1):55-61.

17.Munn Z, Moola S, Lisy K, Riitano D, Tufanaru C. Methodological guidance for systematic reviews of observational epidemiological studies reporting prevalence and cumulative incidence data. Int J Evid Based Healthc. 2015;13(3):147-53.

18.Schwarzer G, Chemaitelly H, Abu-Raddad LJ, Rücker G. Seriously misleading results using inverse of Freeman-Tukey double arcsine transformation in meta-analysis of single proportions. Research synthesis methods. 2019.

19.Rücker G, Schwarzer G, Carpenter JR, Schumacher M. Undue reliance on I 2 in assessing heterogeneity may mislead. BMC medical research methodology. 2008;8(1):79.

20.Borenstein M, Higgins JP, Hedges LV, Rothstein HR. Basics of meta-analysis: I2 is not an absolute measure of heterogeneity. Research synthesis methods. 2017;8(1):5-18.

21.Guyatt GH, Oxman AD, Kunz R, Vist GE, Falck-Ytter Y, Schünemann HJ. What is "quality of evidence" and why is it important to clinicians? BMJ. 2008;336(7651):995.

22.Guyatt GH, Oxman AD, Vist GE, Kunz R, Falck-Ytter Y, Alonso-Coello P, et al. GRADE: an emerging consensus on rating quality of evidence and strength of recommendations. BMJ. 2008;336(7650):924.

23.Iorio A, Spencer FA, Falavigna M, Alba C, Lang E, Burnand B, et al. Use of GRADE for assessment of evidence about prognosis: rating confidence in estimates of event rates in broad categories of patients. BMJ: British Medical Journal. 2015;350:h870.

24.Munn Z, Aromataris E, Tufanaru C, Stern C, Porritt K, Farrow J, et al. The development of software to support multiple systematic review types: the Joanna Briggs institute system for the unified management, assessment and review of information (JBI SUMARI). International journal of evidencebased healthcare. 2019;17(1):36-43. 
25.Stern C, Munn Z, Porritt K, Lockwood C, Peters MD, Bellman S, et al. An international educational training course for conducting systematic reviews in health care: the Joanna Briggs Institute's comprehensive systematic review training program. Worldviews on Evidence-Based Nursing. 2018;15(5):401-8.

\section{Tables}

Table 1: Main characteristics of included systematic reviews $(n=235)$ 


\begin{tabular}{|c|c|}
\hline Number of naming authors & $\begin{array}{l}\text { Median IQR: } 5 \text { (4-7) } \\
\text { Range: 1-18 }\end{array}$ \\
\hline $\begin{array}{l}\text { Protocol registry or } \\
\text { publication* }\end{array}$ & $\begin{array}{l}\text { PROSPERO register: } 51(21.7 \%) \\
\text { Protocol published in peer-reviewed journal: } 5 \text { (2.1\%) } \\
\text { Not reported: } 182(77.4 \%)\end{array}$ \\
\hline Use of a reporting guideline* & $\begin{array}{l}\text { PRISMA: } 161(68.5 \%) \\
\text { MOOSE: } 27(11.5 \%) \\
\text { GATHER: } 2(0.8 \%) \\
\text { Not reported: } 65(27.7 \%)\end{array}$ \\
\hline External funding source & $\begin{array}{l}\text { Yes: } 106(45.1 \%) \\
\text { No: } 73(31.1 \%) \\
\text { Not reported: } 56(23.8 \%)\end{array}$ \\
\hline Number of databases searched & $\begin{array}{l}\text { Median IQR: } 4 \text { (3-6) } \\
\text { Range: 1-14 }\end{array}$ \\
\hline Databases searched* & $\begin{array}{l}\text { MEDLINE: } 231(98.3 \%) \\
\text { Embase: } 146(62.1 \%) \\
\text { Web of Science: } 93(39.6 \%) \\
\text { CENTRAL: } 70(29.8 \%) \\
\text { Scopus: } 72(30.6 \%) \\
\text { CINAHL: } 61(26 \%)\end{array}$ \\
\hline Search strategy presented & $\begin{array}{l}\text { Full search strategy presented for at least one } \\
\text { database: } 159(67.6 \%) \\
\text { Only presented terms used in the search: } 69 \text { (29.4\%) } \\
\text { Nor reported: } 7(3.0 \%)\end{array}$ \\
\hline $\begin{array}{l}\text { Number of studies included in } \\
\text { the review }\end{array}$ & $\begin{array}{l}\text { Median (IQR): } 24 \text { (15-41.5) } \\
\text { Range: 2-1147 }\end{array}$ \\
\hline $\begin{array}{l}\text { Quality assessment of individual } \\
\text { studies* }\end{array}$ & $\begin{array}{l}\text { JBI: } 21(8.9 \%) \\
\text { JBI (adapted): } 5 \text { (2.1\%) } \\
\text { Hoy, } 2012: 10(4.3 \%) \\
\text { Hoy, } 2012 \text { (adapted): } 7 \text { (3.0\%) }\end{array}$ \\
\hline
\end{tabular}




\begin{tabular}{|c|c|}
\hline & $\begin{array}{l}\text { Loney, } 1998: 6 \text { (2.6\%) } \\
\text { Loney, } 1998 \text { (adapted): } 2 \text { (0.9\%) } \\
\text { Newcastle-Ottawa Scale: } 10 \text { (4.3\%) } \\
\text { Newcastle-Ottawa Scale (adapted): } 13 \text { (5.5\%) } \\
\text { Downs and Black (adapted): } 2 \text { (0.9\%) } \\
\text { STROBE: } 15 \text { (6.4\%) } \\
\text { STROBE (adapted): } 7 \text { (3.0\%) } \\
\text { New tool (not adaptation) specific for the review: } 24 \\
\text { (10.2\%) } \\
\text { Non-validated tool, used by a similar review } \\
\text { previously: } 24 \text { (10.2\%) } \\
\text { Others: } 92 \text { (39.1\%) } \\
\text { Not conducted: } 47 \text { (20\%) }\end{array}$ \\
\hline Quality of the body of evidence & $\begin{array}{l}\text { GRADE: } 4(1.7 \%) \\
\text { Oxford: } 1(0.4 \%) \\
\text { Mean STROBE score: } 1(0.4 \%) \\
\text { JBI grades of recommendation: } 1(0.4 \%) \\
\text { AHCPR consistency of evidence: } 1(0.4 \%) \\
\text { Not conducted: } 227(96.6 \%)\end{array}$ \\
\hline Data-synthesis & $\begin{array}{l}\text { Qualitative only: } 83 \text { (35.3\%) } \\
\text { Meta-analysis of prevalence data: } 152(64.7 \%)\end{array}$ \\
\hline
\end{tabular}

* Adds to more than $100 \%$ because some reviews were counted in more than one option.

Table 2: Methods used for meta-analysis $(n=152)$ 


\begin{tabular}{|c|c|}
\hline Methods approach & $\begin{array}{l}\text { Classic: } 151(99.3 \%) \\
\text { Bayesian: } 1(0.7 \%)\end{array}$ \\
\hline Model* & $\begin{array}{l}\text { Random-effects: } 141(93.4 \%) \\
\text { Fixed-effects: } 7 \text { (4.6\%) } \\
\text { Other: } 2(1.3 \%) \\
\text { Not reported: } 7 \text { (4.6\%) }\end{array}$ \\
\hline $\begin{array}{l}\text { Variance estimator (for random-effect } \\
\text { metanalysis, } \mathrm{n}=141 \text { ) }\end{array}$ & $\begin{array}{l}\text { DerSimonian and Laird: } 30 \text { (21.3\%) } \\
\text { Hartung-Knapp-Sidik-Jonkman: } 4 \text { (2.8\%) } \\
\text { Restricted maximum-likelihood: } 1 \\
(0.7 \%) \\
\text { Not reported: } 106(75.2 \%)\end{array}$ \\
\hline Transformation & $\begin{array}{l}\text { Freeman-Tukey double arcsine: } 32 \\
(21.1 \%) \\
\text { Logit: } 5(3.3 \%) \\
\text { Log: } 4(2.6 \%) \\
\text { Raw: } 2(1.3 \%) \\
\text { Arcsine: } 1(0.7 \%) \\
\text { Arcsine square roots: } 1(0.7 \%) \\
\text { Not reported: } 107(70.4 \%)\end{array}$ \\
\hline Heterogeneity assessment* & $\begin{array}{l}\text { Subgroup analysis: } 89 \text { (58.6\%) } \\
\text { Meta-regression: } 57(37.5 \%) \\
\mathrm{I}^{2}: 144(94.7 \%) \\
\text { Galbraith plot: } 4(2.6 \%) \\
\text { Other (eg. influence analysis, outliers): } \\
54(35.5 \%)\end{array}$ \\
\hline Publication bias & $\begin{array}{l}\text { Begg's test: } 26(17.1 \%) \\
\text { Egger test: } 54(35.5 \%) \\
\text { Funnel plot: } 56(36.8 \%) \\
\text { Doi plot: } 4(2.6 \%) \\
\text { Trim and fill: } 7(4.6 \%)\end{array}$ \\
\hline
\end{tabular}




\begin{tabular}{|c|c|}
\hline & $\begin{array}{l}\text { LFK index: } 4(2.6 \%) \\
\text { Not reported: } 79(52.0 \%)\end{array}$ \\
\hline Prediction interval & $\begin{array}{l}\text { Yes: } 3(2.0 \%) \\
\text { Not reported: } 149 \text { (98.0\%) }\end{array}$ \\
\hline Software* & $\begin{array}{l}\text { STATA: } 83(54.6 \%) \\
\text { R: } 29 \text { (19.1\%) } \\
\text { Comprehensive Meta-analysis: } 14 \\
(9.2 \%) \\
\text { MetaXL: } 11(7.2 \%) \\
\text { MedCalc: } 5 \text { (3.3\%) } \\
\text { Review Manager: } 3(2.0 \%) \\
\text { Open Metanalyst: } 3(2.0 \%) \\
\text { StatsDirect: } 3(2.0 \%) \\
\text { MedScale: } 1(0.7 \%) \\
\text { Not reported: } 5(3.3 \%)\end{array}$ \\
\hline
\end{tabular}

* Adds to more than $100 \%$ because some reviews were counted in more than one option.

\section{Figures}




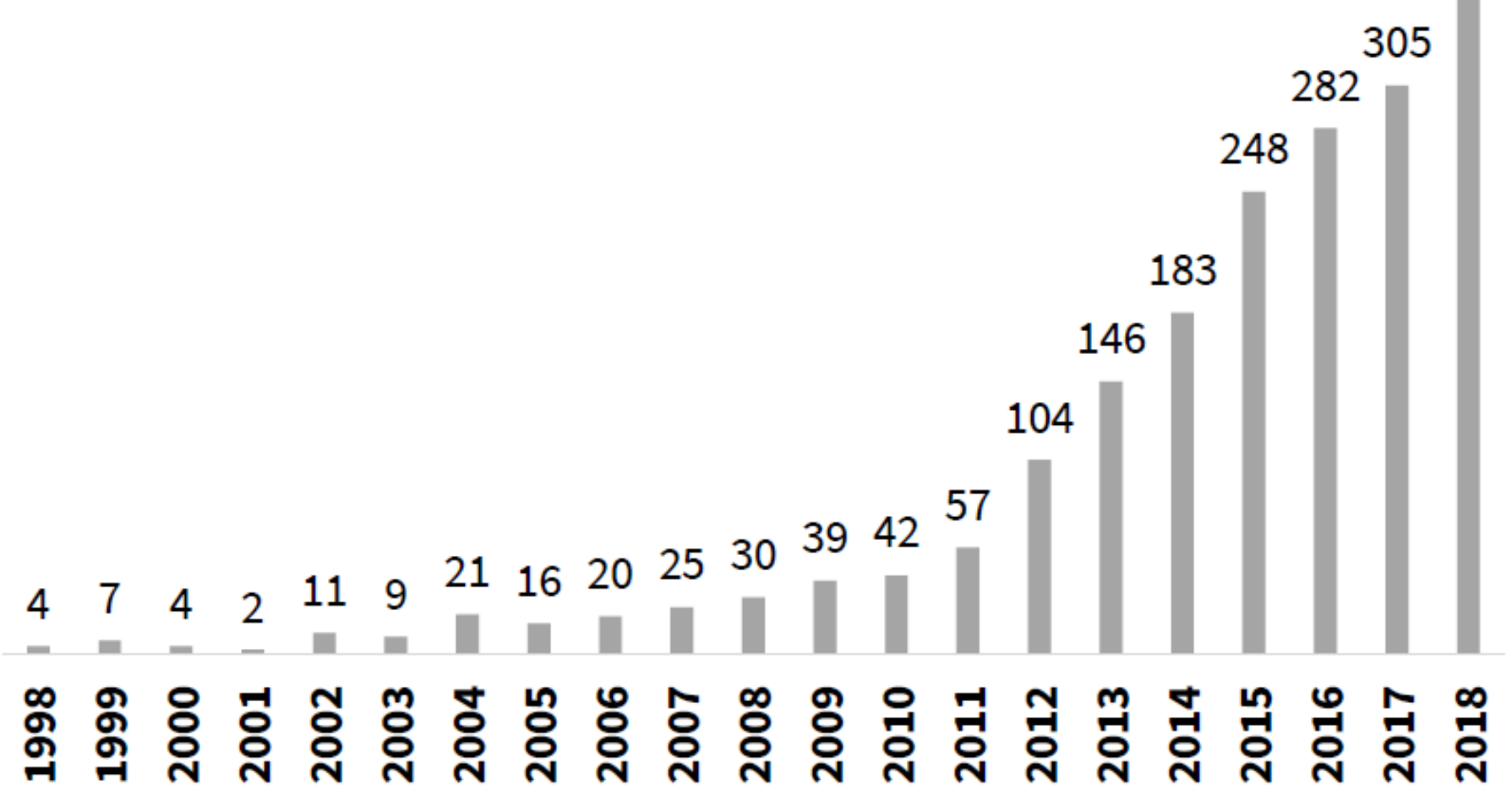

Figure 1

Number of systematic reviews of prevalence indexed in PubMed between 1998 and 2018 


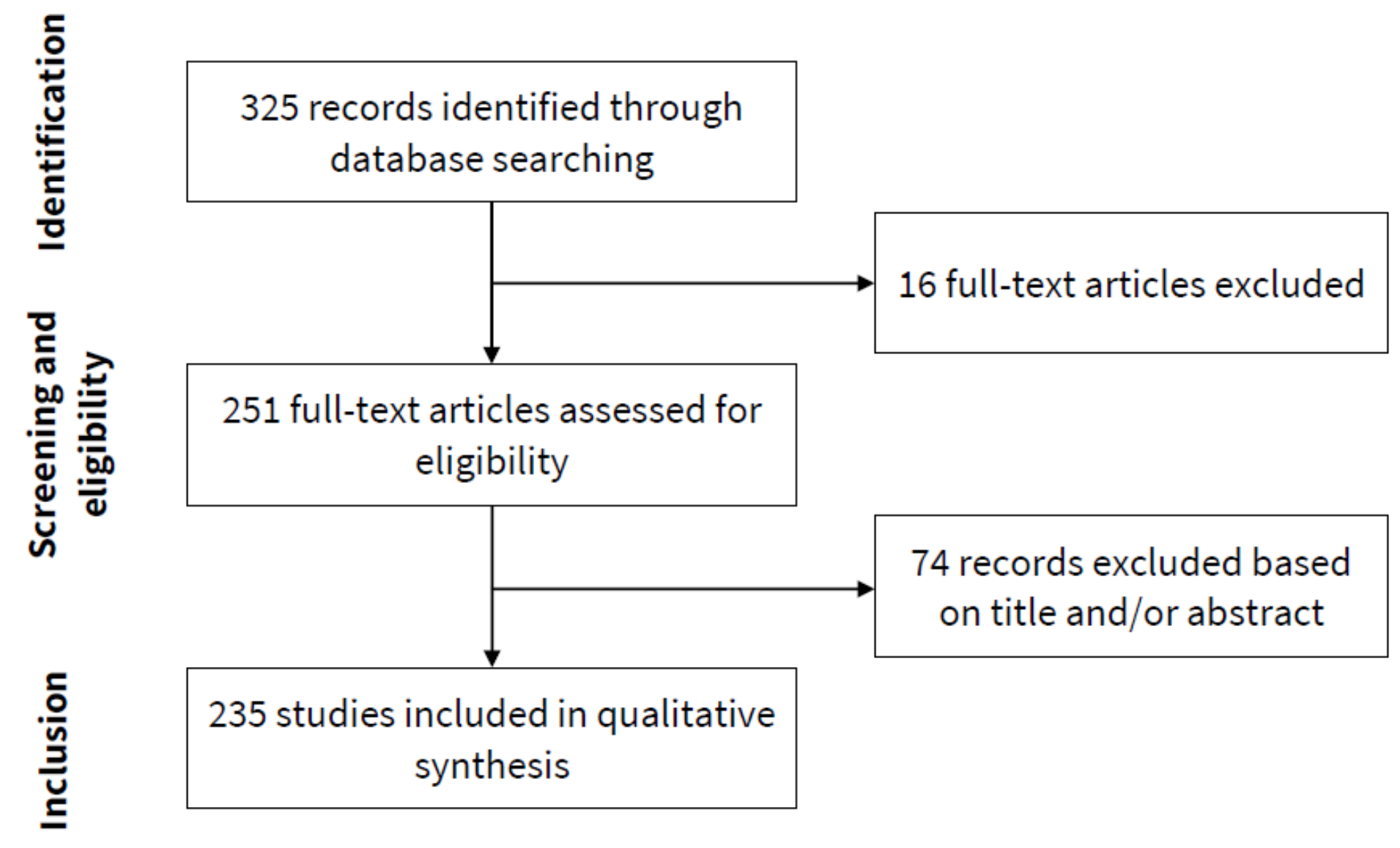

Figure 2

Flowchart of study selection

\section{Supplementary Files}

This is a list of supplementary files associated with this preprint. Click to download.

- Additionalfile1.pdf

- Additionalfile2.pdf

- Additionalfile3.pdf 